\title{
Rotational Speed Measurement Using a Low-Cost Imaging Device and Image Processing Algorithms
}

\author{
Tianyu Wang a, Lijuan Wang ${ }^{\mathrm{b}}$, Yong Yan ${ }^{\mathrm{b}}$, Shuai Zhang ${ }^{\mathrm{a}}$ \\ ${ }^{\text {a }}$ School of Control and Computer Engineering \\ North China Electric Power University, Beijing 102206, China \\ ${ }^{\mathrm{b}}$ School of Engineering and Digital Arts \\ University of Kent, Canterbury, Kent CT2 7NT, U.K.
}

\begin{abstract}
Accurate and reliable measurement of rotational speed is desirable in many industrial processes. A novel method for rotational speed measurement using a low-cost camera and image processing techniques is presented in this paper. Firstly, sequential images are continuously processed using a similarity evaluation method to obtain the periodic similarity level of captured images. Subsequently, the rotational speed is determined from the periodicity of a restructured signal through Chirp-Z transform and parabolic interpolation based autocorrelation, respectively. The measurement principle and system design are presented. The advantages of the proposed measurement system include non-contact measurement, low cost, no markers required and high accuracy. Experimental investigations into the effects of the periodicity detection algorithm, frame rate and image resolution on the accuracy and reliability of the measurement system are conducted on a purpose-built test rig. Experimental results demonstrate that the system with the frame rate of $\mathbf{1 0 0}$ fps yields a measurement error within $\pm 0.6 \%$ over a speed range from 100 to 3000 RPM (Revolutions Per Minute). More accurate and reliable speed measurements over a wider speed range are achievable with higher frame rates.
\end{abstract}

Keywords-rotational speed measurement; image processing; image similarity evaluation; Chirp- $Z$ transform; autocorrelation

\section{INTRODUCTION}

Rotating machineries are widely applied in a range of applications such as energy, power, transportation and manufacturing industries. Since rotational speed reflects the operating conditions, it can serve as an essential parameter for the fault diagnosis and condition monitoring of rotating machineries. There are various tachometers available that utilize different measuring principles for practical applications $[1,2]$. Mechanical tachometers are well-known conventional devices for rotational speed measurement. They are normally mounted on a rotating shaft or contacted with the moving surface. The additional mass to the system may affect the dynamical characteristics of the object being measured and generate wear for long-time operation [3]. The magnetic method is unsuitable for nonmagnetic materials due to the nature of magnetic sensing principle [4]. The measurement accuracy of the electrostatic sensors based system is less satisfactory at a lower speed due to less electrostatic charge generated on the rotor surface [5, 6]. More recently, vibration signal processing techniques have been combined with accelerometers to estimate the instantaneous rotational speed of a rotor $[7,8]$. However, such systems are difficult to obtain accurate measurements in a harsh industrial environment.

In recent years vision-based approaches have been deployed to measure rotational speed in recognition of their advantages of non-contact measurement and simple system structure [9-14]. A rotational speed measurement system using a high-speed camera and a double-sine-varying-density fringe pattern was proposed by Zhong et al. [9]. Kim et al. [10] used a high-speed camera with a switching colour pattern on the rotor for rotational speed measurement. In general, these imaging based systems are implemented through tracking high-contrast markers or speckle patterns on the shaft surface. In order to reduce the effect of motion blur due to marker tracking, highspeed cameras (more than US\$13,000) with a minimum frame rate of $800 \mathrm{fps}$ (frames per second) are usually used to acquire clear images. However, the low affordability of high-speed cameras limits the wide application of such systems. It is therefore desirable to develop techniques that use low-cost imaging devices to achieve satisfactory rotational speed measurements.

A low-cost imaging system for rotational speed measurement through image similarity evaluation and spectral analysis has been reported by Wang et al. [15]. However, a marker is required to be fixed on the cross section of a rotor. This requirement is difficult to meet in some industrial applications. In this paper the imaging system for rotational speed measurement focuses on the side face of the shaft without the use of a marker. Such a system requires lower maintenance and has wider applicability than the marker based systems. Moreover, new methods for periodicity determination based on Chirp-Z transform (CZT) and parabolic interpolation based auto-correlation (PIAC) are proposed to determine the rotational speed from the periodicity of the signal. Comparative assessments between the two methods for periodicity determination are conducted. In addition, the effects of frame rate and image resolution on the performance of the measurement system are quantified and discussed. In comparison with existing high-speed imaging systems, the proposed system is significantly more cost-effective. 


\section{Methodology}

\section{A. Measurement principle}

Fig. 1 shows the fundamental principle of the vision based rotational speed measurement system. An imaging device (Basler acA800-510um at a cost of ca US\$500) is placed perpendicular to the axis of the shaft. In this case, the intensity distribution of the acquired images depends on a variety of factors, including surface properties of the shaft (i.e. surface roughness, natural pattern, etc.), illumination conditions and background. For certain operating and environment conditions, the image intensity distribution is mainly determined by the natural texture of the surface. When a shaft is in rotational motion, the intensity distribution of the images varies with time due to the inherent surface non-uniformity and vibration of the shaft. The rotational speed of the shaft can be measured by determining the periodic patterns in an image sequence. An image similarity evaluation algorithm, i.e. two-dimensional correlation (CORR2), is used to quantify the similarity between the first frame image and the subsequent images. With the continuous processing of the images, the similarity level is regarded as a periodic signal. The periodicity of the reconstructed signal is in fact equal to the time of the shaft rotating for one complete revolution. The periodicity is obtained using periodicity determination methods, CZT or PIAC, in this study. A detailed description of the methods is given in Section II.C.

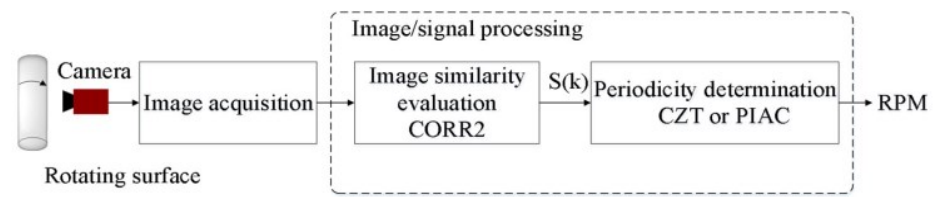

Fig. 1. Principle of the measurement system.

\section{B. Image similarity evaluation}

Two-dimensional correlation (CORR2) is a method for establishing the degree of probability that a linear relationship exists between two measured quantities [16]. In this case, the correlation coefficient indicates the degree of similarity between two images with the same size. For two images $X$ and $Y$ with a resolution of $m \times n$ pixels, the correlation coefficient $\mathrm{r}$ is defined as:

$$
r=\frac{\sum_{i=1}^{m} \sum_{j=1}^{n}[X(i, j)-\bar{X}][Y(i, j)-\bar{Y}]}{\sqrt{\sum_{i=1}^{m} \sum_{j=1}^{n}[X(i, j)-\bar{X}]^{2} \sum_{i=1}^{m} \sum_{j=1}^{n}[Y(i, j)-\bar{Y}]^{2}}}
$$

where $X(i, j)$ and $Y(i, j)$ are the gray-scale values at the point $(i$, $j)$ in the images $X$ and $Y$, respectively. $\bar{X}$ and $\bar{Y}$ are the mean intensity values of the matrices $X$ and $Y$, respectively. The larger the correlation coefficient, the higher the similarity between the two images. In this measurement system, the first acquired image is considered as the reference. With a continuous comparison with the intensity distribution of the first image, an image sequence is transformed to a time-domain signal indicating the similarity level of the sequential images. In other words, the similarity level of the sequential images is normalized to the intensity distribution of the first image. Fig. 2 presents the typical resulting similarity signal at a rotational speed of 1000 RPM. As expected, the signal is periodic due to the rotational motion of the target.

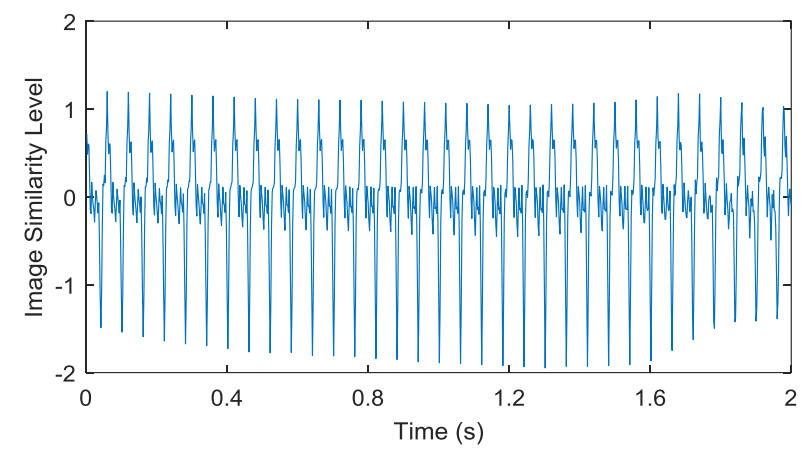

Fig. 2. Typical resulting signal of the image similarity level.

\section{Determination of periodicity}

The rotational speed is obtained from the periodicity of the reconstructed similarity signal (Fig.2) through the use of periodicity determination methods. Due to the low frame rate of a low-cost imaging device, common methods for periodicity determination, such as Fast Fourier Transform and auto-correlation do not perform well with limited sampling time $[17,18]$. In order to improve the measurement accuracy and resolution of the low-cost imaging system, Chirp- $Z$ transform (CZT) and parabolic interpolation based autocorrelation (PIAC) are deployed and compared in this study.

\section{1) $C Z T$}

The main frequency of the reconstructed signal indicating the similarity level, $f$, is equal to the rotational frequency, which is determined through CZT in this study. The location of the dominate peak is corresponding to the rotational frequency, which is then converted into the rotational speed:

$$
N=60 f
$$

CZT is an effective frequency determination method, which computes the frequency spectrum over a certain frequency band in a more detailed way to achieve highresolution frequency measurement [17]. This method is capable of exploring the peak response of a signal within a smaller band of frequencies than the total Nyquist frequency spectrum and significantly refining the low-resolution Fourier spectrum with low computational complexity. Given a sequence with a finite length $S(k)(k=1, \ldots, N)$ of the similarity signal, the Chirp-Z transform $R(m)$ of $S(k)$ is defined as

$$
R(m)=\sum_{k=1}^{N} S(k) Z_{M}^{m k}(m=0,1, \ldots, M-1)
$$

where $M$ is the number of sampling points in the Chirp-Z transform with any possible positive integer value. $Z_{M}^{m k}$ is the transformation kernel and is calculated as 


$$
Z_{M}^{m k}=\exp \left\{-j \frac{2 \pi k}{f_{s}}\left[f_{0}+\frac{\left(f_{1}-f_{0}\right) m}{M}\right]\right\}
$$

where $f_{s}$ is the sampling rate and $\left[f_{0}, f_{1}\right]$ is the frequency observation interval, which is the priori information about the rough range of the actual rotational frequency. In this study, $f_{0}$ and $f_{1}$ are given, respectively, by

$$
\begin{aligned}
& f_{0}=f_{F}-\frac{f_{s}}{N} \\
& f_{1}=f_{F}+\frac{f_{s}}{N}
\end{aligned}
$$

where $f_{\mathrm{F}}$ is determined from the frequency with the highest amplitude in the frequency spectrum of the signal by applying the Fast Fourier Transform algorithm.

Once the Chirp-Z transform $R(m)$ is obtained, the maximum amplitude of the high-resolution frequency spectrum corresponds to the main frequency component of the similarity signal $S(k)$, which is regarded as the rotational frequency $f$.

\section{2) PIAC}

The period of the signal of the similarity level $S(k)$ $(k=1, \ldots, N)$ is equal to the period of the rotational motion. The period can be estimated from the auto-correlation function of the reconstructed signal. A normalized auto-correlation function is defined as

$$
R_{a}(m)=\frac{\sum_{k=1}^{N} S(k) S(k+m)}{\sum_{k=1}^{N} S^{2}(k)}
$$

where $N$ is the number of images in similarity evaluation and is the number of points in the correlation computation. $m$ $(m=0 \ldots, N)$ is the number of delayed points. The normalized auto-correlation function is used to obtain correlation coefficient, namely value of the dominate peak (other than the unity at $m=0$ ). The location of the peak on the time axis of auto-correlation function is the coarse rotation period.

However, the period may not be located at the discrete points in the correlation function. In the measurement system using a low-cost camera, the performance of the autocorrelation algorithm is limited by a relatively low frame rate (below 500fps), which can lead to significant errors in period estimation. Therefore, an effective interpolation strategy, i.e. parabolic interpolation of the peak in the auto-correlation function is employed to generate a closer approximation to the actual rotational period. This interpolation method determines a fine peak by fitting a parabola through three sampling points around the coarse peak [19]. This approach has been commonly used in time delay estimation due to high accuracy and low computational complexity. In this case, the abscissas of three samples $\mathrm{P}_{1}\left(x_{1}, y_{1}\right), \mathrm{P}_{2}\left(x_{2}, y_{2}\right)$ and $\mathrm{P}_{3}\left(x_{3}, y_{3}\right)$ used for parabolic interpolation, which are located on the time axis of the auto-correlation function with the corresponding ordinates being the correlation coefficient, are obtained from equations, respectively

$$
\begin{aligned}
& x_{1}=\tau-\frac{1}{f_{s}} \\
& x_{2}=\tau \\
& x_{3}=\tau+\frac{1}{f_{s}}
\end{aligned}
$$

where $\tau$ is the coarse estimate of the rotational period determined from the auto-correlation function. $f_{s}$ is the frame rate. The parabola that fits these points is obtained from

$$
y=a x^{2}+b x+c
$$

with the parabola coefficients as follows

$$
\begin{gathered}
a=\frac{\left(x_{1}-x_{3}\right)\left(y_{1}-y_{2}\right)-\left(x_{1}-x_{2}\right)\left(y_{1}-y_{3}\right)}{\left(x_{1}-x_{2}\right)\left(x_{1}-x_{3}\right)\left(x_{2}-x_{3}\right)} \\
\mathbf{b}=\frac{\left(y_{1}-y_{2}\right)-a\left(x_{1}-x_{2}\right)^{2}}{\left(x_{1}-x_{2}\right)} \\
c=y_{1}-a x_{1}^{2}-b x_{1}
\end{gathered}
$$

Consequently, the fine rotational period $\mathrm{T}$, represented by the abscissa of the obtained parabola's vertex, is determined from

$$
T=-\frac{b}{2 a}
$$

The rotational speed $N$ is finally determined from the fine rotational period $\mathrm{T}$ :

$$
N=\frac{60}{T}
$$

\section{EXPERIMENTAL RESULTS AND DISCUSSION}

\section{A. $\quad$ Test rig}

Fig. 3 shows the experimental setup of the rotational speed measurement system. The shaft is made of stainless steel with a diameter of $60 \mathrm{~mm}$, connecting with an $\mathrm{AC}$ servo motor through a rigid coupling. The rotational speed is adjustable from 0 to 3000 RPM. The camera focuses on the side face of the shaft. A strip-shaped LED light source is employed to control the illumination conditions. A laptop is used to control the camera and store the image data from the camera through a USB 3.0 cable. Fig. 4 illustrates a typical image of the shaft captured by the low-cost imaging device. Image similarity evaluation, signal processing, including Chirp-Z transform and parabolic interpolation based auto-correlation, and rotational speed calculation are realized in the laptop. In this study a commercial laser based tachometer (UNI-T, model UT372) with a strip-shaped reflective tape on the cross section of the shaft is used to obtain an independent reference to assess the accuracy of the measured speed from the proposed system. The best achievable accuracy of the reference tachometer stated in the operation manual is $\pm 0.04 \%$. 


\section{B. Test program}

To evaluate the effects of relevant factors on the measurement results, three series of experimental tests, as outlined in Table I, were conducted on the test rig. A comparison of the two periodicity determination algorithms was carried out in Test I. In order to study the effects of frame rate and image resolution on the performance of the measurement system, different frame rates $(100 \mathrm{fps}, 300 \mathrm{fps}$ and $500 \mathrm{fps})$ and image resolutions $(640 \times 480,320 \times 240$, $160 \times 120,80 \times 60$ pixels) of the imaging device were assessed in Tests II and III, respectively. In this study measurement accuracy is represented in terms of relative error between the measured speed and the reference speed. The repeatability of the system for a given condition is represented in terms of normalized standard deviation of the measured speed. A total of 30 measurements under the same test condition were recorded for each repeatability test.

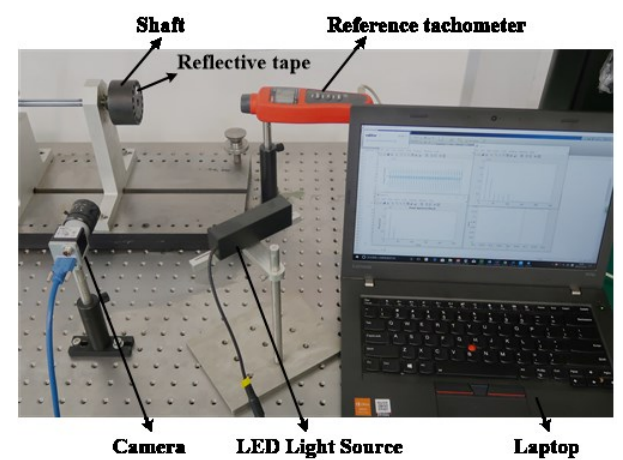

Fig. 3. Experimental setup of the rotational speed measurement system.

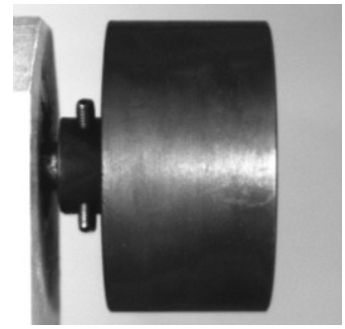

Fig. 4. Typical image of the rotating shaft.

Table I. Test Programme

\begin{tabular}{ccccc}
\hline No & $\begin{array}{c}\text { Signal } \\
\text { processing } \\
\text { algorithm }\end{array}$ & Frame rate (fps) & $\begin{array}{c}\text { Image resolution } \\
\text { (pixels) }\end{array}$ & Speed (RPM) \\
\hline I & CZT/PIAC & 500 & $640 \times 480$ & \\
II & CZT & $100 / 300 / 500$ & $640 \times 480$ & $100-3000$ \\
& & 100 & $640 \times 480,320 \times 240$, & \\
III & CZT & & $160 \times 120,80 \times 60$ & \\
& & & & \\
\hline
\end{tabular}

\section{Comparison between CZT and PIAC}

In order to compare the performance of the periodicity determination algorithms, a series of experimental tests was carried out under the same conditions using CZT and PIAC. Fig. 5 depicts a comparison between the measured and the reference speeds over the range of 100-3000 RPM. It is evident that the measured speed is very close to the reference reading with a maximum error of $\pm 0.65 \%$ for either algorithm. As shown in Fig. 5, the two algorithms yield a similar trend. The relative error and normalized standard deviation decrease with rotational speed. For instance, for PIAC algorithm, the relative error is around $0.65 \%$ at the speed of 100 RPM with the normalized standard deviation of about $0.7 \%$, while the error is significantly reduced to within $\pm 0.1 \%$ when the rotational speed increases. This outcome is also consistent with observations in other sets of experiments due to the fact that more rotating periods are available for signal processing at a higher speed during the same sampling time. It is also worth noting that CZT performs better than PIAC for the same speed in terms of accuracy and repeatability. At the speed of 100 RPM, the relative error of CZT is within $\pm 0.4 \%$ and the normalized standard deviation is around $0.4 \%$, whereas the relative error is around $0.65 \%$ for PIAC. The reason for this difference in performance is that the low frame rate of the camera results in the low resolution of the auto-correlation function and hence uncertainty in the sampling points for the parabolic interpolation of the peak in the auto-correlation function. Therefore, CZT algorithm is employed for periodicity determination in the following study.

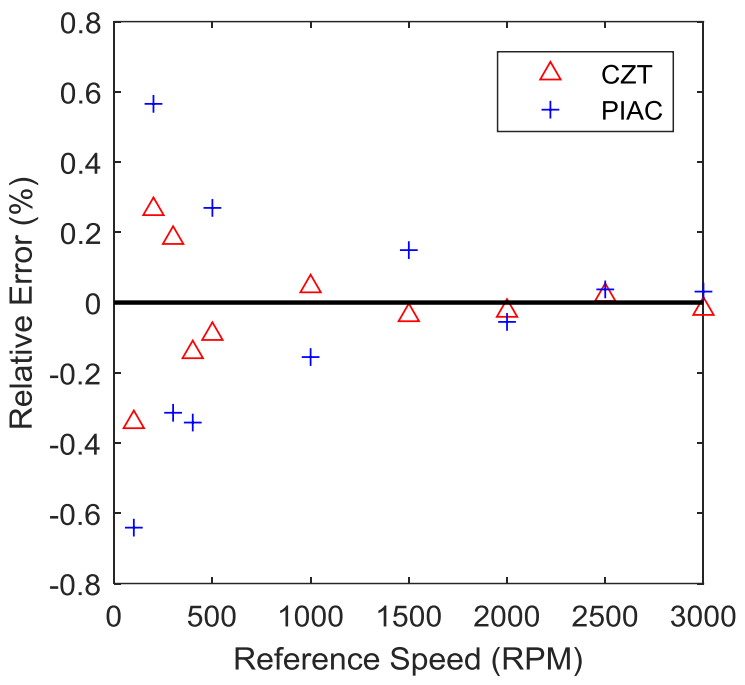

(a) Relative error

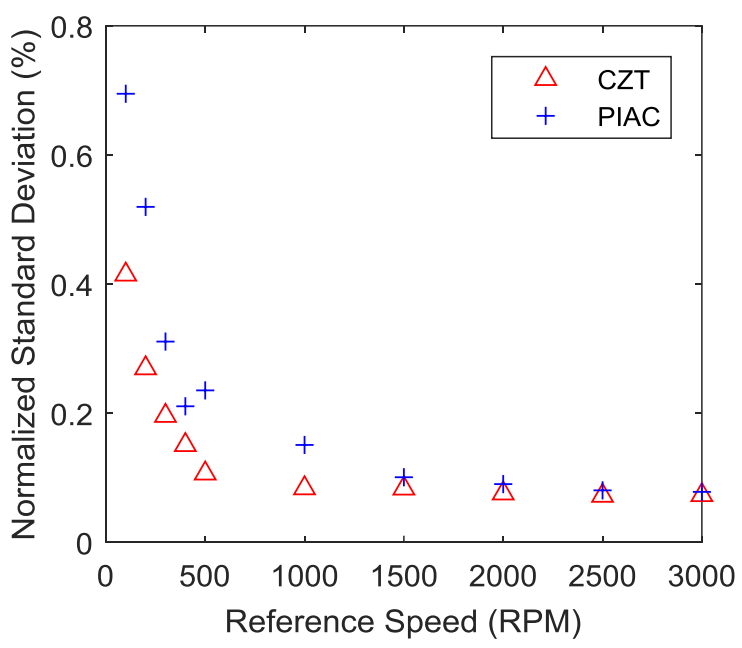

(b) Normalized standard deviation

Fig. 5. Comparison of similarity signal processing algorithms. 


\section{Effects of frame rate}

The frame rate is an important factor for the proposed system. A series of experimental tests was conducted with $100 \mathrm{fps}, 300$ fps and $500 \mathrm{fps}$, respectively, to quantify the effects of the frame rate. As shown in Fig. 6, the comparison between the measured and reference speeds demonstrates that the proposed system, even with the frame rate of $100 \mathrm{fps}$, is capable of providing accurate and reliable measurement over the whole speed range. The relative error is within $\pm 0.6 \%$ whilst the normalized standard deviation is no greater than $0.6 \%$ under all test conditions. This result verifies the feasibility and potential of a low-cost camera with a low frame rate for rotational speed measurement. In addition, the accuracy and repeatability are both improved as the rotational speed increases. It is important to note that the frame rate limits the measurement range of the rotational speed. In order to avoid temporal aliasing, the frame rate of the camera should be at least twice the rotational frequency according to the Nyquist sampling criterion. In other words, the maximum measurable speed for a given frame rate $\left(f_{s}\right)$ is $\left(30 \times f_{s}\right)$ RPM. As expected, a higher sampling frequency generates more accurate and repeatable results due to the smoother signal with the increased sampling points. Consequently, a higher frame rate is essential for higher measurement accuracy and better repeatability as well as extending the measurable speed range.

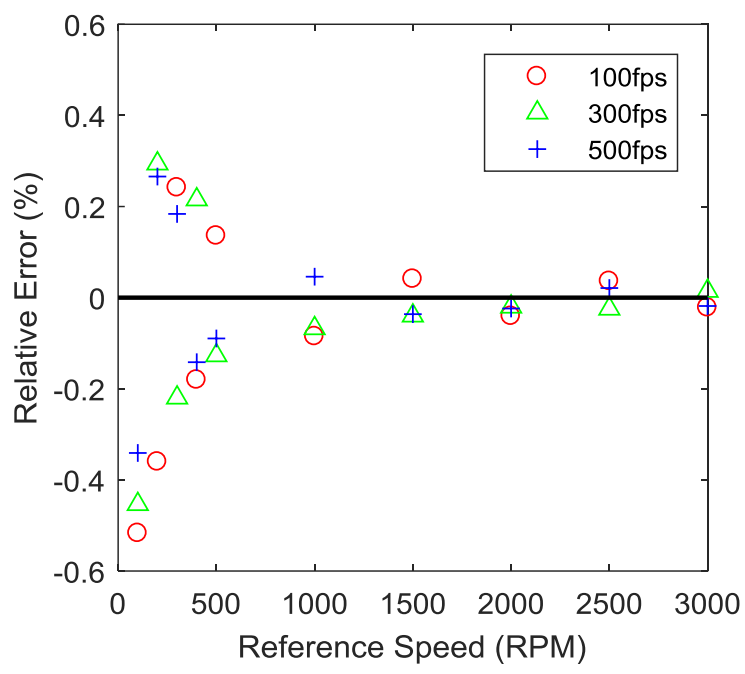

(a) Relative error

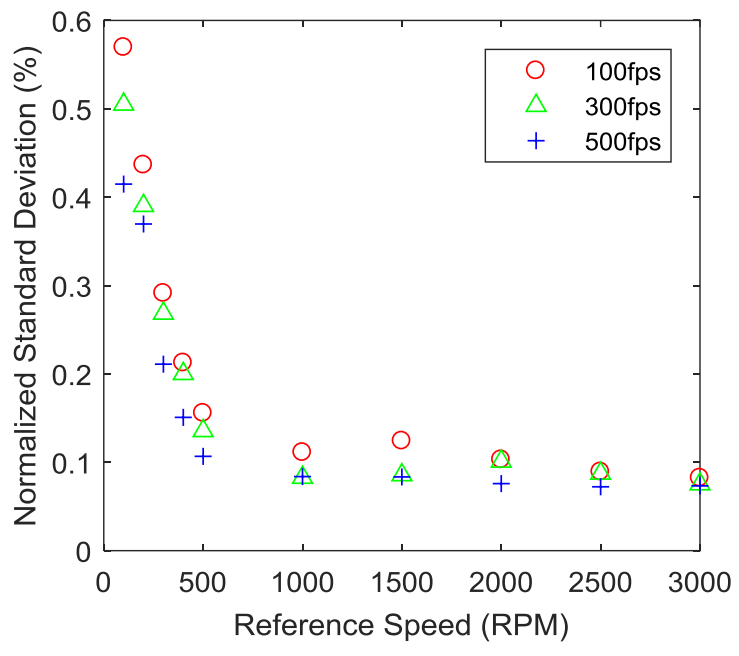

(b) Normalized standard deviation

Fig. 6. Effects of different frame rates.

\section{E. Effects of image resolution}

As a key specification of the imaging system, image resolution is crucial for the measurement performance. The imaging device in this measurement system provides a range of image formats with different resolutions. In order to study the effects of image resolution, different resolutions, including $640 \times 480,320 \times 240,160 \times 120$ and $80 \times 60$ pixels, were tested. Fig. 7 indicates that the rotational speed can be measured accurately and reliably even when the image resolution is as low as $80 \times 60$ pixels. Under this circumstance the measurement system has a shorter computational time and hence fast system response. As expected, the captured images with the highest resolution outperforms all other image formats. This outcome is attributed to the fact that an increasing volume of pixel data is used to sense the changes in the intensity distribution due to the rotational motion of the shaft, enhancing the periodicity of the similarity signal.

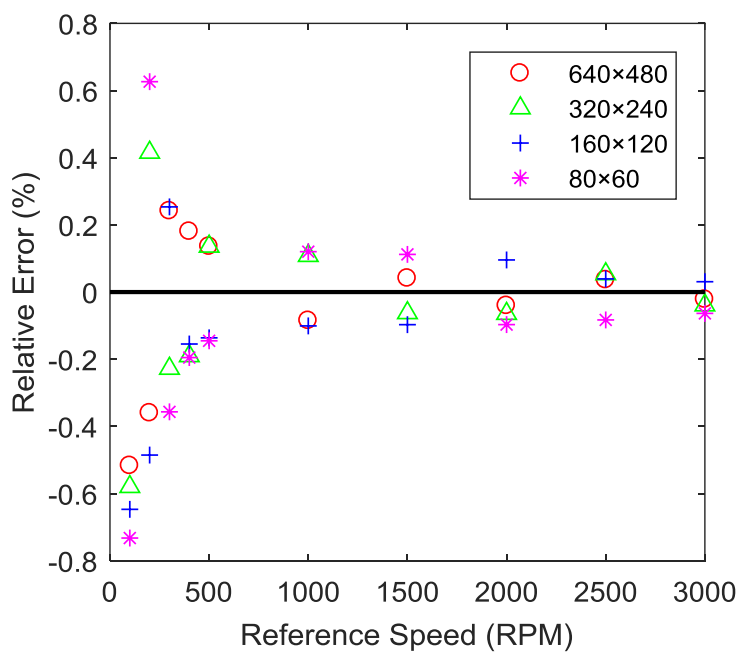

(a) Relative error 


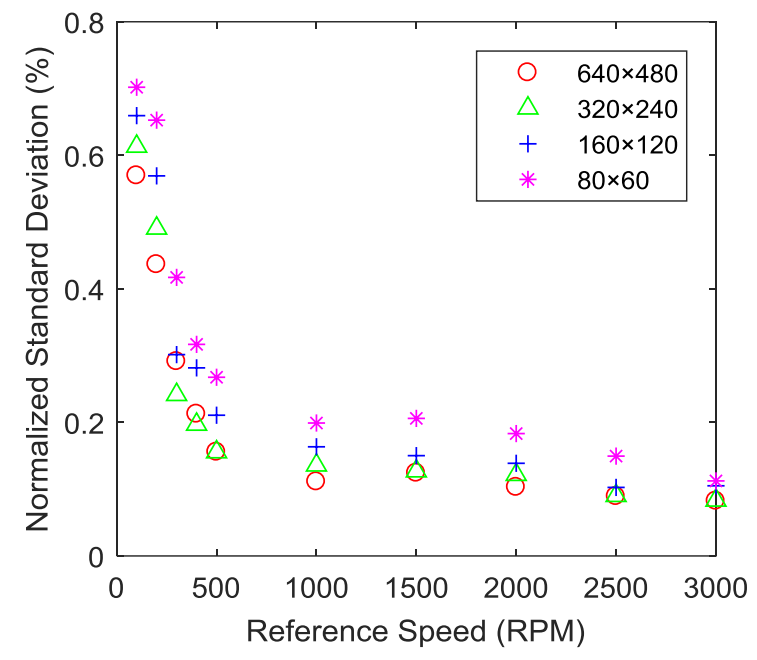

(b) Normalized standard deviation

Fig. 7. Effects of different image resolutions.

\section{CONCLUSIONS}

Experimental results under a range of controlled test conditions have suggested that the rotational speed measurement system based on a low-cost imaging device performs well in terms of accuracy and repeatability without the use of any markers on the object in motion. The low-cost camera with a frame rate of 100 fps yields a relative error of no greater than $\pm 0.6 \%$ over the speed range from 100 to 3000 RPM. For rotational speeds over 1000 RPM and given a sufficient frame rate and image resolution, both the measurement error and the normalised standard deviation are consistently within $\pm 0.1 \%$. The results presented have indicated that the system performance is still satisfactory even if the image resolution is as low as $80 \times 60$. It has been found that CZT is a more suitable algorithm for periodicity determination than PIAC under a low frame rate. Further work will focus on the investigations into other relevant factors that may affect the measurement system, including shooting angle, exposure time and illumination conditions as well as field trials of the system.

\section{ACKNOWLEDGEMENTS}

The authors wish to acknowledge the National Natural Science Foundation of China (No. 51375163), the Chinese Ministry of Education (No. B13009), the Fundamental Research Funds for the Central Universities (No. 2017XS157) for providing financial support for this research. Shuai Zhang would like to thank the IEEE Instrumentation and Measurement Society for 2016 Graduate Fellowship Award.

\section{REFERENCES}

[1] A. Segovia, G. Mayra, L. C. Longoria and A. Diaz, "Stroboscopic microcontroller-based tachometer," Rev. Sci. Instrum., vol. 70, no. 3, pp. 1875-1879, 1999

[2] R. Myers, R. A. Islam, M. Karmarkar and S. Priya, "Magnetoelectric laminate composite based tachometer for harsh environment applications," Appl. Phys. Lett., vol. 91, no. 12, pp. 122904-1-122904-3, 2007.

[3] Y. S. Didosyan, H. Hauser, H. Wolfmayr, J. Nicolics and P. Fulmek, "Magneto-optical rotational speed sensor," Sens. Actuators A, Phys., vol. 106, nos. 1-3, pp. 168-171, 2003.

[4] Y. Li, F. Gu, G. Harris and A. Ball, "The measurement of instantaneous angular speed,” Mech. Syst. Signal. Pr., vol. 19, pp. 786-805, 2005.

[5] L. Wang, Y. Yan, Y. Hu and X. Qian, "Rotational speed measurement through electrostatic sensing and correlation signal processing," IEEE Trans. Instrum. Meas., vol. 63, no. 5, pp. 1190-1199, 2014.

[6] L. Wang, Y. Yan, Y. Hu and X. Qian, "Rotational speed measurement using single and dual electrostatic sensors," IEEE Sens. J., vol. 15, no. 3, pp. 1784-1793, 2015.

[7] J. Urbanek, T. Barszcz and J. Antoni, "A two-step procedure for estimation of instantaneous rotational speed with large fluctuations," Mech. Syst. Signal Pr., vol. 38, no. 1, pp. 96-102, 2013.

[8] H. Lin and K. Ding, "A new method for measuring engine rotational speed based on the vibration and discrete spectrum correction technique," Measurement," vol. 46, no. 7, pp. 2056-2064, 2013.

[9] J. Zhong, S. Zhong, Q. Zhang and Z. Peng, "Measurement of instantaneous rotational speed using double sine-varying-density fringe pattern,” Mech. Syst. Signal Pr., vol. 103, no. 3, pp. 117-130, 2018.

[10] H. Kim, Y. Yamakawa, T. Senoo and M. Ishikawa, "Visual encoder: robust and precise measurement method of rotation angle via high-speed RGB vision,” Opt. Express., vol. 24, no. 12, pp. 13375-13386, 2016.

[11] X. Zhang, J. Chen, Z. Wang, N. Zhan and R. Wang, "Digital image correlation using ring template and quadrilateral element for large rotation measurement," Opt. Lasers Eng., vol. 50, no. 7, pp. 922-928, 2012.

[12] T. Suzuki, H. Nakamura, O. Sadaki and J. Greivenkamp, "Smallrotation-angle measurement using an imaging method," Opt. Eng., vol. 40, no. 3, pp. 426-432, 2001.

[13] J. Guo, C. Zhu, S. Lu, D. Zhang and C. Zhang, "Vision-based measurement for rotational speed by improving Lucas-Kanade template tracking algorithm," Appl. Optics., vol. 55, no. 25, pp. 7186-7194, 2016.

[14] X. Zhu and S. Yu, "Measurement angular velocity based on video technology," in Proc. of Int. Congress on Image and Signal Processing (CISP), , pp. 1936 - 1940, Shanghai, China, 15-17 Oct 2011.

[15] Y. Wang, L. Wang and Y. Yan, "Rotational speed measurement through digital imaging and image processing," in Proc. IEEE Int. Instrum. Meas. Technol. Conf., pp. 260-265, Torino, Italy, 22-25 May 2017.

[16] A. Kaur, L. Kaur and S. Gupta, "Image recognition using coefficient of correlation and structural similarity index in uncontrolled environment," Intel. J. Comput. Appl., vol. 59, no. 5, 2012.

[17] D. Granados-Lieberman, R. J. Romero-Troncoso, E. Cabal-Yepez, R. A Osornio-Rios and L. A. Franco-Gasca, "A real-time smart sensor for high-resolution frequency estimation in power systems," Sensors, vol. 9, no. 9 , pp. 7412-7429, 2009

[18] W. Zhang, C. Wang and Y. Wang, "Parameter selection in crosscorrelation-based velocimetry using circular electrostatic sensors," IEEE Trans. Instrum. Meas., vol. 59, no. 5, pp. 1268-1275, 2010.

[19] R. Moddemeijer, "On the determination of the Position of Extrema of Sampled Correlators," IEEE Signal Process. Mag., vol. 39, no. 1, pp. 216-219, 1991. 